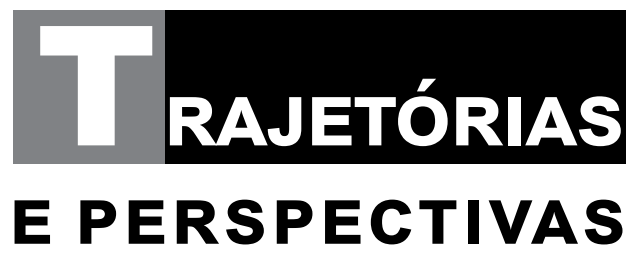




\section{Arthur Ramos (1903-1949): chefe do Departamento de Ciências Sociais da Unesco}

\section{Arthur Ramos (1903-1949): head of the Unesco Department of Social Sciences}

\section{Amurabi Oliveira}

Brasil. Universidade Federal Fluminense. Doutor em Sociologia pela Universidade Federal de Pernambuco (UFPE). Professor da Universidade Federal de Santa Catarina (UFSC). Pesquisador do CNPq. ID ORCID: https://orcid.org/0000-0002-7856-1196.E-mail:amurabi_cs@hotmail.com.

\section{Resumo}

Arthur Ramos (1903-1949) foi um dos principais antropólogos brasileiros do século XX, tendo sido um dos principais agentes na institucionalização das ciências sociais no Brasil. Apesar de ter tido uma carreira relativamente curta, por causa da sua morte prematura, ele foi um dos pioneiros na circulação internacional de pesquisadores brasileiros nas ciências sociais. Foi professor visitante nos Estados Unidos e chefe do Departamento de Ciências Sociais da Unesco. Neste artigo, examino a atuação de Arthur Ramos nessa instituição, apontando para sua inserção internacional, que possibilitou que ele fosse indicado para esse cargo, e os principais planos que ele demonstrava ter naquele momento. Reafirmo aqui seu pioneirismo na circulação acadêmica internacional, e aponto de que forma suas ideias perduraram na instituição mesmo após sua morte.

Palavras-chave: Arthur Ramos, História da Antropologia, Circulação Internacional.

\section{Abstract}

Arthur Ramos (1903-1949) was one of the main Brazilian anthropologists of the twentieth century, having been one of the main agents in the institutionalization of social sciences in Brazil. Despite having a relatively short career, given his untimely death, 
he was one of the pioneers in the international circle of Social Sciences Brazilian researchers, having been a visiting professor at the United States and head of Unesco's Department of Social Sciences. In this article I examine the work of Arthur Ramos in this institution, noting his international connections which allowed him to be nominated for this position, and the main plans he seemed to have at that time. I reaffirm here his pioneering spirit in the international academic circles, and point out how his ideas endured in the institution even after his death.

Keywords: Arthur Ramos, History of Anthropology, International Circulation.

\section{INTRODUÇÃO}

Arthur Ramos (1903-1949) foi um dos mais importantes antropólogos brasileiros da primeira metade do séculoXX. Foi professor titular de Antropologia na Faculdade Nacional de Filosofia, espaço no qual desenvolveu um importante papel na rotinização do conhecimento antropológico (OLIVEIRA, 2019a), além de fundador da Sociedade Brasileira de Antropologia e Etnologia (SBAE), primeira sociedade científica no campo da antropologia no Brasil (AZEREDO, 1986).

Seus diálogos intelectuais ultrapassavam as barreiras nacionais, o que era realizado por meio de uma ampla rede de contatos que desenvolveu ao longo dos anos, especialmente no que diz respeito aos intelectuais que estavam dedicados aos estudos do "problema do negro" no Novo Mundo. Figuras como Fernando Ortiz (1881-1969), Rüdiger Bilden (1893-1980), Melville Herskovits (1895-1963), Roger Bastide (1898-1974), Donald Pierson (1900-1995) etc., faziam parte de sua ampla rede de contatos, com os quais mantinha distintos graus de proximidade e parceria.

Apesar dessa posição singular no campo acadêmico, dada sua inserção internacional num momento no qual as ciências sociais brasileiras ainda estavam institucionalizando-se, e seu protagonismo nos estudos afro-brasileiros (MOTTA, 2016), pode-se afirmar que só em período mais recente o trabalho de Ramos tem sido revisitado com mais intensidade 
(BARROS, 2011; CAMPOS, 2004). Certamente, parte desse "ostracismo relativo" deveu-se às próprias disputas em torno da narrativa acerca da história das ciências sociais no Brasil, que afirma o modelo uspiano como um marco no processo de produção de determinado padrão de cientificidade (MICELI, 1989), relegando autores e instituições que fogem (ou antecedem) desta construção ao status de "pré-científicos" ou "provincianos" (REESINK; CAMPOS, 2014).

Viso neste artigo, sobretudo, contribuir para o debate em torno da história da antropologia no Brasil, e, de forma articulada, visibilizar a circulação internacional da antropologia brasileira, apontando-a como um fenômeno que não é recente. O recorte escolhido para tanto foi a curta atuação de Arthur Ramos como diretor do Departamento de Ciências Sociais da Organização das Nações Unidas para a Educação, a Ciência e a Cultura (Unesco) em 1949, ano de sua morte, o que tento recuperar através de cartas e documentos diversos que compõem o "Arquivo Arthur Ramos" depositado na Biblioteca Nacional. Minha intenção é compreender como foi possível sua inserção naquele espaço, e apontar para algumas ações que ele planejava executar estando a frente desse cargo.

Para uma melhor compreensão de minha análise por parte dos leitores, dividirei meu trabalho em mais três seções: a) na primeira, farei um breve apanhado do processo de circulação de Arthur Ramos por diferentes espaços institucionais, evidenciando sua circulação pelo Brasil e exterior; b) posteriormente, procurarei visibilizar como ele foi construindo uma rede de contatos e parcerias no exterior que possibilitou uma maior visibilidade de seu trabalho; c) por fim, analiso sua breve atuação como o primeiro diretor do departamento de Ciências Sociais da Unesco, visibilizando os planos que ele possuía em termos de atuação naquela instituição.

\section{ARTHUR RAMOS, UM ANTROPÓLOGO EM CIRCULAÇÃO}

Ramos nasceu no interior de Alagoas, na cidade de Pilar, filho do médico dr. Manoel Ramos de Araújo Pereira e Dona Ana Ramos de Araújo Pereira, 
numa família inserida nos círculos das elites culturais locais (BARROS, 2008). A escolha por continuar na profissão familiar o levou à tradicional Faculdade de Medicina da Bahia em 1921, na qual doutorou-se em Ciências Médicas em 1926 com a tese O primitivo e a loucura, recebendo o título de doutor em Ciências Médicas no mesmo ano.

Na escrita de sua tese, Ramos recorreu principalmente aos trabalhos de Sigmund Freud (1856-1939) e de Lucien Lévy-Bruhl (1987-1939), que perduram como principais referências na tese de livre docência, Sordície nos alienados: ensaio de uma psicopatologia da imundice, defendida na mesma faculdade. Passou então a atuar no Serviço Médico Legal do estado da Bahia, onde tem os primeiros contatos com as questões da cultura negra, através do material etnográfico acumulado por Raimundo Nina Rodrigues (1862-1906).

Em que pese as diferenças e mesmo rupturas existentes entre o trabalho de Ramos e o de Nina Rodrigues, aquele amiúde se afirmava continuador da “Escola Nina Rodrigues”. Segundo Corrêa (2013, p. 155):

\begin{abstract}
A incompatibilidade entre a produção intelectual dos discípulos e a de seu proclamado mestre se expressa não só na transformação de certos elementos de definição, de certos recortes teóricos utilizados por Nina Rodrigues, com um sentido que eles não poderiam ter tido, como também no fato de que essa transformação, isolando-os de sua época e de seu contexto teórico, alterava a relação que cada um desses elementos ou recortes mantinha com outros originalmente, alterando também o seu sentido. $\mathrm{O}$ que eles fazem é retomar certos temas tratados por Nina Rodrigues - e por vários outros intelectuais de sua época e redefini-los a seus próprios termos, ou nos de sua própria época, arranjando-os dentro de outro sistema de relações.
\end{abstract}

Nesse sentido, a reafirmação exaustiva de Ramos como continuador da obra de Nina Rodrigues - o que incluiu a organização e publicação de material de "seu mestre" - parece estar inserida de forma mais contundente na busca por legitimação de Ramos no campo acadêmico, primeiramente nos "estudos do negro", e posteriormente de forma disciplinar na antropologia, o 
que implicava também a afirmação de Nina Rodrigues como um predecessor da antropologia brasileira.

Em que pese ter alcançado bastante notoriedade na Bahia, destacando-se o fato de ser percebido como um dos pioneiros na introdução do debate da psicanálise no Brasil (ABRÃO, 2011; GARCIA, 2014), foi com sua ida ao Rio de Janeiro que sua carreira acadêmica se consolidou. Essa mudança é realizada em 1933, assumindo cargo no Serviço de Ortofrenia e Higiene Mental a pedido de Anísio Teixeira (1900-1971), que nesse momento era secretário de Educação na prefeitura do Distrito Federal ${ }^{1}$, permanecendo no cargo até 1939. Ainda nesse ínterim, ele foi designado em 1934 e assumiu em 1935 em caráter interino a cátedra de Psicologia Social na Universidade do Distrito Federal (UDF), publicando em 1936 o livro Introdução à psicologia social, que era fruto de seu curso.

É no contexto da UDF que Ramos passa a ter contato com Gilberto Freyre (1900-1987), que também havia se tornado professor da UDF a pedido de Teixeira, e naquele momento ocupava ainda o cargo de diretor do Departamento de Ciências Sociais (CAMPOS, 2004).

Em 1939, Ramos passou a ocupar o cargo de professor interino na área de Antropologia e Etnografia. Ao ocupar o espaço da cátedra, ele passa a legitimar sua posição como médico-antropólogo, transitando de uma área para a outra, num movimento que não era incomum para o período. 1939 é um ano emblemático, na medida em que a UDF foi dissolvida e incorporada à Universidade do Brasil (UB), dentro do contexto das reformas conduzidas por Gustavo Capanema (1900-1985). O corpo docente e discente da UDF fora incorporado à Faculdade Nacional de Filosofia (FNF) da UB.

Como bem demonstra Fávero (2008), a formação do corpo docente da FNF esteve envolta em meio às disputas acadêmicas e políticas no nível local e nacional. Obviamente que o campo acadêmico é continuamente movido por elementos "extra-acadêmicos" (BOURDIEU, 2011); todavia, dada a

Importante marcar que parte significativa das mudanças que vinham sendo implementadas por Anísio Teixeira nesse período vinculavam-se às ideias difundidas pela chamada "Escola Nova", de modo que a existência de tal seção representava, justamente, um dos aspectos da modernização no sistema educacional almejado nesse período. 
proximidade com o governo federal, isso ficava ainda mais evidente no caso da FNF. Havia inúmeras cartas nas quais se recomendavam distintos nomes para assumir as cátedras existentes; alguns nomes eram mais, outros menos recorrentes; o de Ramos, por exemplo, fora recomendado por Heloísa Alberto Torres (1895-1977), na época diretora do Museu Nacional (LIPPI DE OLIVEIRA, 1995), que depois integrou o júri do concurso para professor titular em 1945.

Além dessa mudança dentro do campo acadêmico brasileiro, Ramos passou paulatinamente a ampliar seus espaços de circulação, ultrapassando as barreiras nacionais, chegando a ocupar o cargo de Special Lecturer in Sociology no departamento de sociologia da Universidade do Estado da Louisiana na década de 1940. Essa maior circulação será possível a partir do capital social que Ramos passou a acumular nos anos posteriores, o que será objeto de maior apreciação na seção seguinte.

\section{A FORMAÇÃO DE REDES INTERNACIONAIS}

A partir da década de 1930, passou a existir uma ampla rede de colaboração entre pesquisadores brasileiros e norte-americanos, especialmente no que tange aos estudos das relações raciais. Ramos, que fora organizador com Edison Carneiro (1912-1972) do II Congresso Afro-Brasileiro, realizado em Salvador em 1937, era uma das figuras centrais nesse processo (SANSONE, 2012).

Como indicado na seção anterior, é na UDF que Ramos entra em contato com Freyre, que àquele tempo já mantinha uma considerável rede de relações com pesquisadores norte-americanos, uma vez que realizara sua graduação na Universidade de Baylor, no Texas, seu mestrado na Universidade de Colúmbia, em Nova Iorque, e havia sido ainda professor visitante em Stanford, no Departamento de História Social. Um dos pesquisadores americanos com o qual Freyre possuía maior proximidade era Bilden² (PALLARES-BURKE, 2012), que o apresentou a Ramos, como indica a correspondência de Bilden para Ramos datada de 11 de dezembro de 1936:

2 Bilden era em verdade alemão, porém radicado nos Estados Unidos. 
Eu também sou grato ao nosso mútuo amigo Gilberto Freyre por ter feito possível este contato. Quando eu voltar novamente ao Brasil, como eu espero fazer em um futuro próximo, eu procurarei mais adiante encontrá-lo pessoalmente aproveitando diretamente da sua opinião e conselhos especializados (BILDEN, 1936, tradução nossa) ${ }^{3}$.

Bilden estava em contato com grandes nomes da antropologia norte-americana, como Franz Boas (1858-1942) e Ruth Benedict (1887-1948), bem como outros nomes que gravitavam em torno desses dois. Nesse sentido, poderíamos dizer que a Universidade de Colúmbia constituiu um espaço privilegiado de formação de ciclos sociais, que por sua vez envolviam pesquisadores brasileiros e norte-americanos (OLIVEIRA, 2017). Bilden fora o responsável por apresentar Herskovits, que havia sido orientando de Boas, aos pesquisadores brasileiros, o que incluía Ramos (YELVINGTON, 2007).

Certamente, Herskovits fora o pesquisador estrangeiro com o qual Ramos manteve o diálogo mais intenso, trocando cartas e materiais por um longo período de tempo (GUIMARÃES, 2004), além de ter frequentado seu curso sobre aculturação nos Estados Unidos.

Também foi Bilden que introduziu por cartas Ruth Landes (1908-1991) a Ramos, e este por sua vez apresentou Landes a Edison Carneiro. O jornalista e pesquisador baiano veio a ser seu principal parceiro no Brasil para a elaboração de sua pesquisa, posteriormente publicada em 1947 com o título de The city of women, e vinte anos depois em português com o título $A$ cidade das mulheres, em edição devidamente revisada por Carneiro. Notadamente, nesse caso houve também inúmeras tensões, com o questionamento a posteriori por parte de Herskovits e de Ramos acerca das conclusões apresentadas por Landes (OLIVEIRA, 2019b).

Foi também no contexto da UDF que Ramos conheceu Pierson, que havia frequentado o curso de sociologia ministrado por Freyre. Tal como Bilden e

\footnotetext{
No original: "Also I am grateful to our mutual friend Gilberto Freyre for making possible this contact. When I came again to Brazil, as I hope to do in the near future, I look foward to meeting you personally and profitting directly from your expert opinion and advice".
} 
Landes, Pierson havia permanecido por um período na Universidade de Fisk ${ }^{4}$, de modo que havia também um partilhamento de referências em comum, em termos de amizades e de contatos acadêmicos.

Além do papel relevante que Pierson veio a ter no Brasil, tornando-se professor da Escola Livre de Sociologia e Política de São Paulo, foi ele que apresentou por cartas Ramos a Thomas Lynn Smith (1903-1976), que era professor do departamento de Sociologia da Universidade do Estado da Louisiana. Partiu de Smith o convite para que Ramos ministrasse um curso sobre "Raças e Culturas no Brasil", o que após diversas negociações se efetivou entre o final de 1940 e início de 1941. Essa experiência foi profundamente marcante na carreira de Ramos, uma vez que possibilitou que ele estreitasse ainda mais os contatos com pesquisadores norte-americanos. Apesar do fato de ter havido outros convites posteriormente, foi apenas nessa ocasião que ele teve uma experiência acadêmica mais longa nos Estados Unidos.

Concomitante a essas atividades, Ramos movimentava-se intensamente em torno da institucionalização da antropologia no Brasil, o que incluiu a SBAE. Estar à frente dessa sociedade também o possibilitou entrar em contato com outras sociedades científicas internacionais; nesse momento, ele não era unicamente professor de antropologia da FNF, mas também presidente da única sociedade científica no campo da antropologia no Brasil.

Os contatos com pesquisadores caribenhos também foram relevantes nesse período, com destaque para as correspondências trocadas com o cubano Ortiz, que na época (final dos anos 1930) era presidente da Sociedad de Estudios Afrocubanos, e com o etnógrafo e psiquiatra haitiano Louis PriceMars (1876-1969). A existência dessa rede de contatos, situados não apenas em países hegemônicos no campo da antropologia, aponta que a grande preocupação de Ramos era forjar uma rede de colaboração principalmente entre aqueles que estavam dedicados ao estudo do "negro no Novo Mundo", ou seja, seu crivo era antes temático que disciplinar.

\footnotetext{
A Universidade de Fisk é uma universidade negra localizada no Tenessi, e nos três casos seus respectivos orientadores recomendaram que estes pesquisadores permanecessem um tempo nessa instituição como uma espécie de "estágio preparatório" para as pesquisas sobre relações raciais no Brasil.
} 


\section{DO RIO DE JANEIRO A PARIS: O DEPARTAMENTO DE CIÊNCIAS SOCIAIS DA UNESCO}

O desdobrar da década de 1940 levou a uma ascensão progressiva da carreira de Ramos, marcada não só por suas experiências no exterior e na fundação de uma sociedade científica nacional, mas também pelo acontecimento de ter se tornado professor titular de Antropologia e Etnografia em 1945 na FNF.

Ao que tudo indica por meio dos documentos consultados, foi Paulo Carneiro (1901-1982), que foi embaixador do Brasil nas Nações Unidas entre 1946 e 1958, em articulação com o intelectual mexicano James Torres Bodet (19021974), que foi diretor-geral da Unesco entre 1948 e 1952, o principal articulador da ida de Ramos para o Departamento de Ciências Sociais da Unesco. Apesar de estar aparentemente entusiasmado com o convite, em telegrama datado de 23 de maio de 1949 Ramos indica que necessitaria ao menos de mais dois meses, após a chegada do convite oficial, para regularizar seus compromissos e partir para Paris.

O convite formal para atuar como diretor do Departamento de Ciências Sociais da Unesco chega num telegrama de Bodet datado de 12 de junho de 1949, no qual indica um pagamento anual de 9.200 dólares mais $1.500 \mathrm{em}$ indenizações para alocação pessoal. Ramos responde prontamente por meio de outro telegrama, aceitando o convite e aguardando maiores instruções.

Em 20 de junho de 1949, Bodet envia um novo telegrama, indicando que o contrato será de dois anos. Ele ainda realiza a seguinte ponderação:

Eu não sugeriria que você fizesse um grande esforço, que sem dúvida estaria envolvido em sua saída do Brasil tão cedo, se não fosse o fato de que terei muita necessidade urgente de sua ajuda e colaboração antes e durante a próxima sessão de a Conferência Geral, que começa em 19 de setembro. Se você me informar a data em que poderia sair do Rio e o número de pessoas que o acompanharão, seu transporte será organizado a partir daqui. Sugiro que você me envie essas informações, para que as providências possam ser tomadas com bastante antecedência (BODET, 1949, tradução nossa) ${ }^{5}$.

\footnotetext{
No original: "I would not suggest that you make a great effort, which would undoubtedly be involved in your leaving Brazil so soon, if it were not for the fact that I shall have very urgent need of your help and collaboration before and during the next session of the General Conference, which begins on 19
} 
A tônica desse telegrama aponta para certa urgência para o início das atividades de Ramos. Todavia, aparentemente seu afastamento da FNF não era um trâmite tão simples e rápido, tendo em vista que ele escreve para Carneiro pedindo que explicasse a James Bodet as dificuldades encontradas para seu licenciamento da UB. O fato é que ao final de julho de 1940 Carneiro escreve para Ramos indicando que seu apartamento já estava reservado.

Ramos preocupou-se não apenas com as atividades a serem desenvolvidas em Paris, como também com a continuação de suas atividades no Brasil. Seu pedido para o afastamento foi aprovado na sessão do Conselho Universitário da UB em 14 de julho de 1949, conforme indica a carta datada de 3 de agosto de 1949 de Pedro Calmon (1902-1985), então reitor da universidade, na qual ressalta ser este um fato altamente honroso para a cultura brasileira.

Também ele fora profundamente cuidadoso com relação à continuação das atividades da cátedra de Antropologia na UB. Em carta datada de 4 de agosto de 1949, endereçada ao ministro da Educação e Saúde, Clemente Mariani (1900-1981), Ramos agradece a simpatia e compreensão demonstradas pela sua escolha para dirigir o Departamento de Ciências Sociais da Unesco, e reitera seu desejo de continuar seus estudos sobre o negro, indicando Marina Vasconcellos (1912-1973) para substituí-lo na universidade (RAMOS, 1949d).

Como é sabido, Vasconcellos foi pupila de Ramos, tendo integrado o curso de aperfeiçoamento em Antropologia e Etnografia organizado por Ramos no início da década de 1940, no qual ela apresentou em 1942 a monografia intitulada Movimentos contra-aculturativos no Brasil: Palmares. Ela já havia se tornado a substituta oficial de Ramos em 1941, assumindo suas turmas quando ele estava nos Estados Unidos, e de fato ela fora a primeira mulher a lecionar no curso de Ciências Sociais da FNF (MIGLIEVICH-RIBEIRO, 2015). Apesar de Heloísa Alberto Torres ter demonstrado interesse em ocupar a cátedra de Antropologia e Etnografia durante o período no qual Ramos estaria em Paris, dirigindo o Departamento de Ciências Sociais da Unesco, ele foi categórico

September. If you will let me know the date on which you would be able to leave Rio and the number of persons who will be accompany you, your transportation will be arranged from here. I suggest that you cable this information to me, so that arrangements can be made well in advance". 
em afirmar que a cátedra não estaria vaga, uma vez que Vasconcellos era sua substituta (BARROS, 2008).

A indicação para esse cargo é motivo de bastante orgulho para Ramos, como podemos ver nas cartas que ele escreve para seus amigos e colaboradores informando de sua indicação. Ao prefeito do Distrito Federal, Ângelo Mendes de Morais (1894-1990), ele escreve em 7 de julho de 1949, a Smith em 25 de julho de 1949, e a Bastide em 27 de julho de 1949 (RAMOS, 1949a, 1949b, 1949c). E tão pronto se alocou em Paris, logo começou a organizar planos a serem desenvolvidos na nova instituição.

Ramos era essencialmente um acadêmico, e estava bastante empenhado em realizar no âmbito internacional o que havia feito no Brasil: engajar-se na institucionalização das ciências sociais, e da antropologia em particular. Entre os planos que ele estava organizando nessa direção, encontra-se a publicação de um Boletim das Ciências Sociais, que deveria dialogar com um público para além dos especialistas, teria uma tiragem de 4.000 exemplares e seria uma publicação bilíngue. Também previa a publicação de um Anuário das Ciências Sociais, que deveria ter um caráter mais acadêmico, voltado para um público especializado.

Em documento sem data (é apenas indicado que ele havia chegado há uma semana em Paris), intitulado "Perspectives sur le Département des Sciences Sociales", Ramos realiza os seguintes apontamentos:

Eu pude constatar que a Unesco, em seu Departamento de Ciências Sociais, está trabalhando para encorajar três das principais ciências sociais: Economia Política, Sociologia e Ciência Política, sem mencionar a ajuda que pouco pode lhes trazer a Psicologia Social.

A Antropologia Cultural ainda não encontrou seu lugar no programa do Departamento. Certamente, no projeto de "Tensões", são discutidos alguns problemas relacionados a raças e minorias, mas ainda não foi dada atenção constante aos problemas específicos do homem. É por isso que não entendo por que "os estudos comparativos de culturas", com ênfase particular nas culturas indígenas da África, existem no Departamento de Filosofia e Ciências Humanas, e não no Departamento de Ciências Sociais. 
Este estudo deve, na minha opinião, constituir uma das atividades mais importantes do Departamento de Ciências Sociais. Primeiro, um estudo geral das condições de vida e culturas das massas atrasadas em seus respectivos habitats deve ser realizado em todas as partes do mundo; seria então possível estudá-los diante das culturas dominantes. Em outras palavras, um estudo sobre a vida e a cultura de povos não europeus examinados em relação aos elementos determinantes da civilização europeia.

Nesse sentido, uma das questões que, na minha opinião, deve ser colocada pela Unesco em seu Departamento de Ciências Sociais, é a da assimilação e "aculturação" dos povos indígenas e negros do Novo Mundo.

A ação da Unesco nesse sentido deve ser articulada com a atividade já existente de certas instituições, o Instituto Indigenista Interamericano, Cidade do México, o Instituto Afro-Americano, Cuba e outros (RAMOS, 1949g, tradução nossa) ${ }^{6}$.

Essa longa citação aponta para algumas das questões que Ramos considerou mais importante perseguir no Departamento de Ciências Sociais da Unesco, articulando, portanto, um projeto de internacionalização e projeção da antropologia numa das questões que ele considerava mais relevantes no

\footnotetext{
No original: "J'ai ainsi pu constater que l'Unesco, dans son Département des Sciences Sociales, semploye à encourager trois des principales sciences sociales: L'Economie politique, la Sociologie et les Sciences politiqus, sans oublier d'aide que peu leur apporter la Psychologie sociale.

"L'Anthropologie culturelle n’a pas encore trouvé sa place dans le programme de Département. Certes, dans le projet de 'Tensions', on aborde quelques problèmes relatifs aux races et aux minotirés, mais aucune attention soutenue ne sest encore sur les problèmes spécifiques de l'homme. C’est ainsi que je ne compreds pas porquoi 'létudes comparative des cultures', avec une insistance particulière sur les cultures autochtones de l'Áfrique, existe au Département de Philosophie et des Sciences Humaines plutôt que au Département des Sciences Sociales.

"Cette edtude devrait, à mon avis, constituer une des activités les plus importantes du Département des Sciences Sociales. On devrait d'abord procéder a une étude générale sur les conditions de vie et sur les cultures des masses arriérées dans leurs habitats respectifs, pour toutes les parties du monde; il serait ensuite possible de les estudier en faces des cultures dominantes. En autres termes, une étude sur la vie et la culture des peuples non-européens examinée par rapport aux éléments déterminants de la civilisation européene.

"En ce sens, une des questions que devraient, à mon avis, étre posée par l'Unesco dans son Département des Sciences Sociales, est celle de l'assimilation et "l'acculturation" des peuples indiens et noir de Nouveau Monde.

Laction de l'Unesco, dans cette voie, devra éte articulée avec la activité déjà existante de certaines instituitions, l'Instituto Indigenista Interamericano, de Mexico, l'Afro-American Institue, de Cuba, et autres".
} 
mundo contemporâneo: o debate racial. Chama atenção ainda a ideia de articular as atividades da Unesco com instituições situadas na América Latina, projetando assim agentes situados fora dos eixos hegemônicos das ciências sociais.

Ele realizou ainda um levantamento das associações internacionais já existentes no campo das ciências sociais, apontando que já havia àquela data nas seguintes áreas: ciência política, direito comparado, sociologia, economia, psicologia social. Nesse sentido, ele aponta a necessidade de constituição de uma associação internacional de antropologia nos mesmos moldes, e propõe ainda a criação de um conselho internacional de ciências sociais.

Esse seu empenho pôde ser percebido ainda por meio de sua presença na conferência de fundação da Associação Internacional de Sociologia, que ocorreu entre 5 e 11 de setembro de 1949 em Oslo. Apesar de ter havido o convite para que o Brasil enviasse um representante oficial, que seria Fernando de Azevedo (1894-1974), dado o fato de que no Brasil já havia uma sociedade científica nessa área naquele momento (PLATT, 1998), entretanto, como Azevedo informa em carta datada de 29 de agosto de 1949 para Ramos, não seria possível seu comparecimento à conferência. Nesse cenário, o país não contou com uma representação oficial, uma vez que Ramos estava ali representando a Unesco.

Quando escreveu para Jorge Kingston (1901-), então presidente do Departamento de Ciências Sociais da FNF, em carta datada de 12 de setembro de 1949, Ramos fez referência à conferência de Oslo como sua primeira missão oficial relevante no novo cargo. Dentre as possibilidades de cooperação entre o Brasil e o Departamento de Ciências Sociais da Unesco, Ramos realiza o seguinte apontamento:

Outras possibilidades se abrem para a cooperação de cientistas sociais do Brasil e, em geral, da América Latina, com o meu Departamento, aqui na Unesco. Isto, porém, demora um pouco, até que possa apresentar meu novo programa à Conferência geral de Florença, em maio do ano próximo.

Há, porém, um setor de estudos sobre o problema de raças que já foi delineado pelo meu antecessor, Prof. Klinenberg, que permite encarar a possibilidade de pedir cooperação do Brasil, com a convocação 
de uma reunião preliminar, talvês ainda este ano, em dezembro, para definir o conceito de raça em seus aspectos inter-disciplinares. Penso em contar com umas cinco ou seis pessoas de diferentes países e estou envidando esforços para trazer um cientista social do Brasil que aborde os aspectos sociais da raça (RAMOS, 1949e, p. 2).

Preocupa-se ainda nessa carta com a resolução sobre quem ocupará sua cátedra, pois, ao que parece, apesar de sua indicação para que Vasconcellos o substituísse, ainda havia algumas pendências burocráticas; mas em todo caso, em carta datada de 6 de outubro de 1949, Kingston trata de acalmar Ramos, indicando que esta questão seria resolvida em conformidade com as prescrições regimentais (KINGSTON, 1949).

Parece ser preciso indicar que a atuação de Ramos na Unesco dirigia-se a duas principais direções: a institucionalização das ciências sociais no nível internacional, e o desenvolvimento dos estudos raciais, com foco nos estudos africanos. Isso é reforçado pelas correspondências trocadas com Bastide, nas quais fala-se na promoção de um evento que reunisse diferentes africanistas. O sociólogo francês, em carta datada de 21 de abril de 1949, antes de Ramos assumir o cargo, já falava nessa ideia de promover este evento, e indicava que buscaria financiamento da ONU para tanto. Em outra correspondência, data de 29 de outubro de 1949, Bastide sugere que Ramos elabore um plano de trabalho para o estudo dos negros americanos e que peça ajuda financeira à ONU para tanto (BASTIDE, 1949).

Com Luiz Costa Pinto (1920-2002), Ramos também mantém troca de informações sobre suas atividades na Unesco. Costa Pinto lhe escreve em 24 de setembro de 1949 pedindo mais informações sobre a conferência de Oslo, e solicita ainda alguma possibilidade de financiamento pela Unesco para sua pesquisa sobre migrações internas no Brasil. Ramos o responde em 29 de setembro de 1949, indicando que os processos na Unesco são profundamente "lentos e cautelosos", mas que vislumbra alguma mudança com o programa que ele apresentaria na Conferência de Florença em 1950, mas que mesmo assim ele só seria implementado em 1951. Nessa carta, ele também faz referência à divisão criada por Otto Klineberg (1899-1992), indicando a possibilidade de realizar 
uma reunião preliminar em Paris, para a qual indaga a possibilidade de Costa Pinto comparecer como representante da sociologia brasileira (RAMOS, 1949e).

Apesar dos muitos planos para sua atuação na Unesco, Ramos morreu prematuramente em 31 de outubro de 1949. Havia delineado ainda em meados de outubro de 1949 um plano para o incremento das pesquisas antropológicas e sociológicas no Brasil, que visava, dentre outras coisas, dar uma especial atenção ao "estudo dos grupos negro e indígena para a tarefa de sua integração ao mundo moderno", como indica em carta de 14 de outubro de 1949 endereçada a Mariani (RAMOS, 1949f). E como destaca Maio (1999, p. 142):

Em junho de 1950, a 5 a sessão da Conferência Geral da Unesco, realizada em Florença, aprovou a realização de uma pesquisa sobre as relações raciais no Brasil, mas Arthur Ramos, seu idealizador, havia falecido oito meses antes, sem chegar a definir com maiores detalhes o tipo de estudo que tinha em mente. No entanto, é notável que, mesmo sem sua participação no desenho definitivo da investigação, suas preocupações a respeito do Brasil estavam presentes tanto na versão final do Projeto Unesco quanto nos resultados das diversas pesquisas realizadas em seu âmbito.

Essa orientação estava profundamente afinada com o papel que Ramos percebia que poderia assumir as ciências sociais, cuja institucionalização possibilitaria a superação de um fazer mais "literário" dos estudos sobre os grupos raciais (RAMOS, 1948). Isso aponta, portanto, para o fato de que apesar de não ter tido a oportunidade de concluir seus projetos, Ramos lançou fundamentos relevantes para a elaboração de uma ampla agenda de pesquisa no campo das ciências sociais brasileiras.

Fugiria do foco e do escopo deste artigo aprofundar o papel do Projeto Unesco no estudo das relações raciais no Brasil ${ }^{7}$. Sem embargo, é interessante perceber como ele implicou uma reorientação do debate em vigor até aquele momento. Mais que isso, o desenvolvimento desse projeto consolida as redes de colaboração e pesquisa entre cientistas sociais brasileiros e estrangeiros, possibilitando uma intensa internacionalização do debate.

Para uma melhor análise acerca do papel desse projeto no estudo das relações raciais brasileiras, conferir Pereira e Sansone (2007). 


\section{CONSIDERAÇÕES FINAIS}

Como anunciado desde o princípio deste artigo, o objetivo maior aqui apresentado encontra-se na possibilidade de revisita da própria história da antropologia brasileira, centrando a análise em agentes considerados eventualmente "provincianos" ou "pré-científicos", como é o caso de Ramos. Em que pese seu lugar central na institucionalização das ciências sociais brasileiras, seu legado é recorrentemente apontado como "não antropológico" (BARROS, 2008), ou mesmo relegado ao esquecimento (CAMPOS, 2004).

A revisita de sua atuação no Departamento de Ciências Sociais da Unesco nos possibilita vislumbrar de forma mais clara a agenda de atuação de um antropólogo brasileiro inserido internacionalmente, ocupando uma posição ímpar no campo intelectual de seu tempo. E como pôde ser apontado, apesar da breve atuação no órgão, seu trabalho teve repercussões posteriores que impactaram decididamente as ciências sociais, e o campo de estudo das relações raciais em particular.

Apesar de ter falecido antes da realização da Conferência Geral da Unesco de 1950, Ramos deixou pronto o artigo “A questão racial e o mundo democrático" (La question raciale et le monde démocratique), que seria lido nesse evento e que foi publicado no Bulletin-3-4, v. I de 1949 (BARROS, 2008). O artigo reitera suas preocupações acadêmicas e políticas, deixando clara a forma como percebia o lugar das ciências sociais no mundo contemporâneo, em grande medida confluindo com algumas questões já trazidas em As ciências sociais e os problemas de após-guerra (RAMOS, 1944).

Reafirmo assim, ao fim deste breve trabalho, o pioneirismo de Arthur Ramos em termos de circulação internacional da antropologia brasileira, apontando para seu papel não apenas na institucionalização das ciências sociais no Brasil, mas também para sua visibilidade internacional, pautada principalmente a partir dos estudos das relações raciais naquele contexto. 


\section{REFERÊNCIAS BIBLIOGRÁFICAS}

1. ABRÃO, Jorge Luís Ferreira. Arthur Ramos: precursor da psicanálise de crianças no Brasil. In: BARROS, Luitgarde Oliveira Cavalcanti (org.). Arthur Ramos. Rio de Janeiro: Fundação Miguel de Cervantes, 2011. p. 201-229.

2. AZEREDO, Paulo Roberto. Antropólogos e pioneiros: a história da Sociedade Brasileira de Antropologia e Etnologia. São Paulo: Edusp, 1986.

3. BARROS, Luitgarde Oliveira Cavalcanti (org.). Arthur Ramos e as dinâmicas sociais de seu tempo. Maceió: Edufal, 2008.

4. BARROS, Luitgarde Oliveira Cavalcanti (org.). Arthur Ramos. Rio de Janeiro: Fundação Miguel de Cervantes, 2011.

5. BASTIDE, Roger. [Carta]. Destinatário: Arthur Ramos. São Paulo, 21 abr. 1949. Biblioteca Nacional, Arquivo Arthur Ramos, I-35,22,640.

6. BILDEN, Rüdiger. [Carta]. Destinatário: Arthur Ramos. Nova York, 11 dez. 1936. Biblioteca Nacional, Arquivo Arthur Ramos, I-35,23,714.

7. BODET, Jaime Torres. [Carta]. Destinatário: Arthur Ramos. Paris, 20 jun. 1949. Biblioteca Nacional, Arquivo Arthur Ramos, I-35,24,753.

8. BOURDIEU, Pierre. Homo academicus. Florianópolis: EdUFSC, 2011.

9. CAMPOS, Maria José. Arthur Ramos: luz e sombra na antropologia brasileira. Rio de Janeiro: Edições Biblioteca Nacional, 2004.

10. CORRÊA, Mariza. Ilusões de Liberdade: a escola Nina Rodrigues e a Antropologia no Brasil. Rio de Janeiro: Ed. Fiocruz, 2013.

11. FAILlACE, Vera Lúcia Miranda (org.). Arquivo Arthur Ramos: inventário analítico. Rio de Janeiro: Fundação Biblioteca Nacional, 2004.

12. FÁVERO, Maria de Lourdes. Faculdade Nacional de Filosofia: o corpo docentematizes de uma proposta autoritária. Rio de Janeiro: Editora UFRJ, 2008.

13. GARCIA, Ronaldo Aurélio Gimenes. Arthur Ramos e Durval Marcondes: higiene mental, psicanálise e medicina aplicadas à educação nacional (1930-1950). Educação \& Sociedade, Campinas, v. 35, n. 128, p. 951-966, 2014.

14. GUIMARÃES, Antônio Sergio. Comentários à Correspondência entre Melville Herskovits e Arthur Ramos (1935-1941). In: PEIXOTO, Fernando Arêas; PONTES, Heloisa; SCHWARCZ, Lilian Moritz (org.). Antropologias, histórias, experiências. Belo Horizonte: Editora UFMG, 2004. p. 169-197.

15. KINGSTON, Jorge. [Carta]. Destinatário: Arthur Ramos. Rio de Janeiro, 6 out. 1949. Biblioteca Nacional, Arquivo Arthur Ramos, I-35,32,1.572. 
16. LIPPI DE OLIVEIRA, Lúcia. As ciências sociais no Rio de Janeiro. In: MICELI, Sergio (org.). História das ciências sociais no Brasil. São Paulo: Sumaré, 1995. p. 233-307. v. 2.

17. MAIO, Marco Chor. O Projeto Unesco e a agenda das ciências sociais no Brasil nos anos 40 e 50. Revista Brasileira de Ciências Sociais, São Paulo, v. 14, n. 41, p. 141-158, 1999.

18. MICELI, Sergio. Condicionantes do desenvolvimento das ciências sociais. In: MICELI, Sergio (org.). História das Ciências Sociais no Brasil. São Paulo: Vértice, 1989. p. 72-110. v. 1.

19. MigLiEVICH-RIBEIRO, Adélia. Heloísa Alberto Torres e Marina de Vasconcellos: pioneiras na formação das ciências sociais no Rio de Janeiro. Rio de Janeiro: Editora UFRJ, 2015.

20. MOTTA, Roberto. De Nina a Juana: representações da África e do Candomblé. In: LIMA, Ivaldo Marciano de França et al. (org.). África(s), índios e negros. Recife: Bagaço, 2016. p. 255-288.

21. OliVEIRA, Amurabi. Amizades e inimizades na formação dos estudos afro-brasileiros. Latitude, Maceió, v. 11, n. 2, p. 589-617, 2017.

22. OLIVEIRA, Amurabi. Arthur Ramos e a rotinização da antropologia através de seu ensino. Revista Civitas, Porto Alegre, v. 19, n. 3, p. 1-9, 2019a.

23. OLIVEIRA, Amurabi. Ruth Landes (1908-1991) and her understanding of Brazil in The City of Women. Asian Journal of Latin American Studies, Seul, v. 32, n. 3, p. 29-41, $2019 b$.

24. PALLARES-BURKE, Maria Lúcia. O triunfo do fracasso: Rüdiger Bilden, o amigo esquecido de Gilberto Freyre. São Paulo: Editora Unesp, 2012.

25. PEREIRA, Claúdio L.; SANSONE, Lívio (org.). Projeto Unesco no Brasil: textos críticos. Salvador: Edufba, 2007.

26. PINTO, Luiz A. Costa. [Carta]. Destinatário: Arthur Ramos. Rio de Janeiro, 29 set. 1949. Biblioteca Nacional, Arquivo Arthur Ramos, I-36,2,2.201.

27. PLATT, Jeniffer. A brief history of the ISA: 1948-1997. Madrid: International Sociological Association, 1998.

28. RAMOS, Arthur. As ciências sociais e os problemas do após-guerra. Rio de Janeiro: Casa do Estudante do Brasil, 1944.

29. RAMOS, Arthur. Os grandes problemas da antropologia brasileira. Sociologia, [s. l.], v. X, n. 4, p. 213-226, 1948.

30. RAMOS, Arthur. [Carta]. Destinatário: Ângelo Mendes de Morais. Rio de Janeiro, 7 jul. 1949a. Biblioteca Nacional, Arquivo Arthur Ramos, I-35,17,291A. 
31. RAMOS, Arthur. [Carta]. Destinatário: Thomas Lynn Smith. Rio de Janeiro, 25 jul. 1949b. Biblioteca Nacional, Arquivo Arthur Ramos, I-35,19,428.

32. RAMOS, Arthur. [Carta]. Destinatário: Roger Bastide. Rio de Janeiro, 27 jul. 1949c. Biblioteca Nacional, Arquivo Arthur Ramos, I-35,13,34.

33. RAMOS, Arthur. [Carta]. Destinatário: Clemente Mariani. Rio de Janeiro, 4 ago. 1949d. Biblioteca Nacional, Arquivo Arthur Ramos, I-35,17,248.

34. RAMOS, Arthur. [Carta]. Destinatário: Luís Aguiar da Costa Pinto. [s. l.], 29 set. 1949e. Biblioteca Nacional, Arquivo Arthur Ramos, I-35,18,372A.

35. RAMOS, Arthur. [Carta]. Destinatário: Clemente Mariani. Rio de Janeiro, 14 out. 1949f. Biblioteca Nacional, Arquivo Arthur Ramos, I-35,17,248A.

36. RAMOS, Arthur. Perspectives sur le Département des Sciences Sociales. Paris, 1949g. Biblioteca Nacional, Arquivo Arthur Ramos, I-35,20,454A.

37. REESINK, Mísia; CAMPOS, Roberta. A Geopolítica da antropologia no Brasil: ou como a província vem se submetendo ao leito de Procusto. In: CAMPOS, Roberta; SCOTT, Parry; PEREIRA, Fabiana (org.). Rumos da antropologia no Brasil e no mundo: geopolíticas disciplinares. Recife: Ed. UFPE, 2014. p. 55-81.

38. SANSONE, Lívio. Estados Unidos e Brasil no Gantois: o poder e a origem transnacional dos estudos afro-brasileiros. Revista Brasileira de Ciências Sociais, São Paulo, v. 27, n. 79, p. 9-29, 2012.

39. YELVINGTON, Kevin. Melville J. Herskovits e a institucionalização dos estudos afro-americanos. In: PEREIRA, Claúdio L.; SANSONE, Lívio (org.). Projeto Unesco no Brasil: textos críticos. Salvador: Edufba, 2007. p. 149-171. 